\title{
A 13th-century cystic echinococcosis from the cemetery of the Monastery of Badia Pozzeveri (Lucca, Italy)
}

\section{Antonio Fornaciari ${ }^{\mathrm{a}} *$}

antonio.fornaciari@med.unipi.it

Raffaele Gaeta ${ }^{\mathrm{a}}$

Letizia Cavallini

Giacomo Aringhieri

Randa Ishak

Fabrizio Bruschi $^{\mathrm{d}}$

Valentina

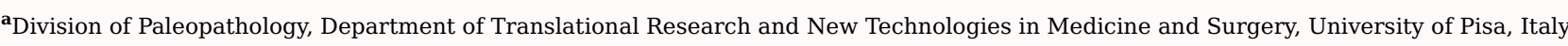

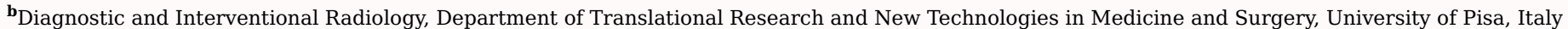

${ }^{\mathbf{c}}$ Department of Civil and Industrial Engineering, University of Pisa, Italy

d Laboratory of Parasitology, Department of Translational Research and New Technologies in Medicine and Surgery, University of Pisa, Italy

${ }^{*}$ Corresponding author at: Department of Translational Research and New Technologies in Medicine and Surgery, University of Pisa. Via Roma 57, 56126, Pisa, Italy.

\section{Abstract}

Objective

To differentially diagnose a calcified formation recovered from a 13th century AD grave from the Tuscan monastery of Badia Pozzeveri, Lucca, Italy. Materials

A calcified formation from the thoraco-abdominal region of a skeleton buried in the monastery cemetery.

Methods

Cone Beam Computed Tomography, Scanning Electron Microscope and Energy Dispersive X-Ray Spectroscopy. Results

A hollow, calcified ovoid formation was identified as typical of a hydatid cyst, permitting the diagnosis of cystic echinococcosis in a 35-45-year-old female.

\section{Conclusions}

The study reveals the circulation of the parasite Echinococcus granulosus in the region of Lucca in late medieval Tuscany.

\section{Significance}

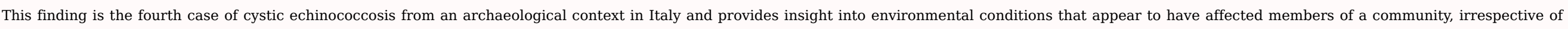
social status.

Limitations

Caution and the application of multiple analyses must be exercised in the differential diagnosis to discriminate among calcified formations.

Suggestions for further research 
Keywords: Hydatid Cyst; Paleoparasitology; Calcification; Funerary Archaeology; Medieval monastery; Zoonosis

\section{Introduction}

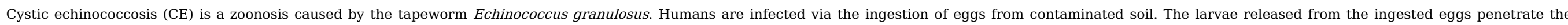

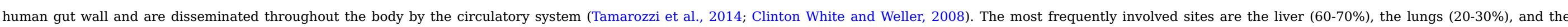

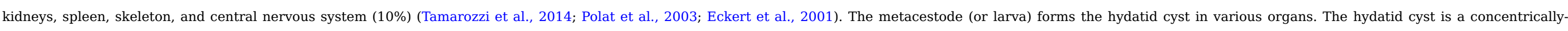

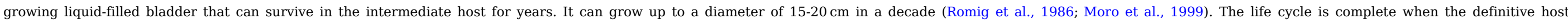

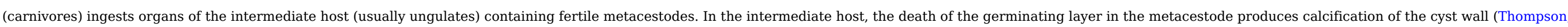

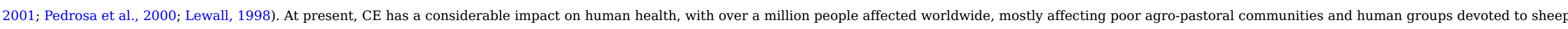

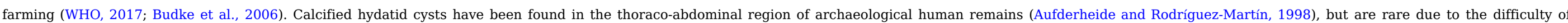
recognizing and recovering them and their fragility.

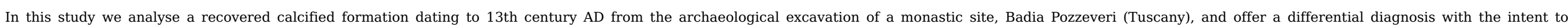
understand health and disease in this region of Italy.

\section{Material and methods}

\subsection{Historical background}

The archaeological site of Badia Pozzeveri is located approximately $10 \mathrm{~km}$ southeast of the city of

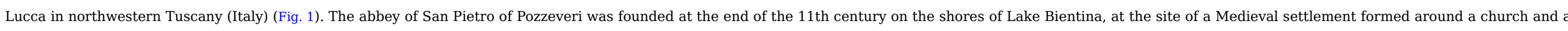

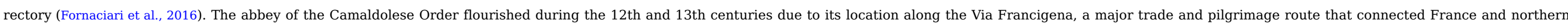

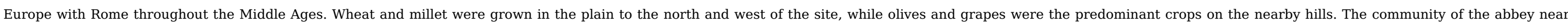

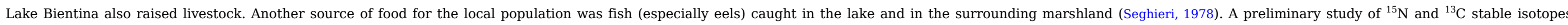
suggests that individuals buried in the cemetery consumed a diet of meat and vegetables (Amaro et al., 2019).

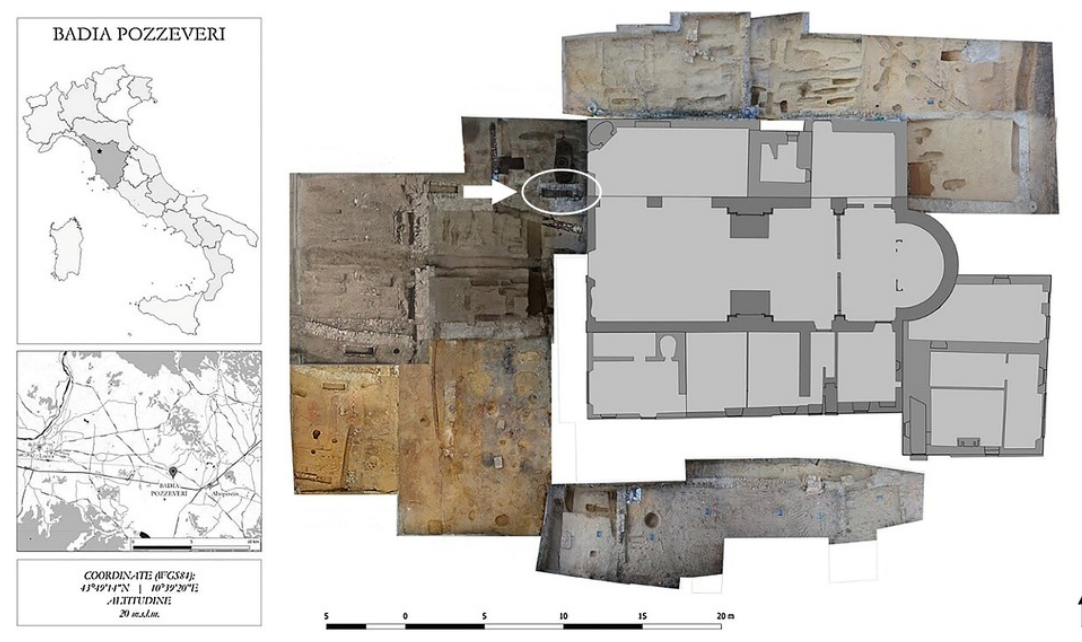




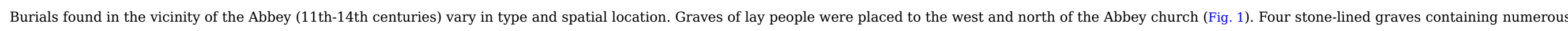

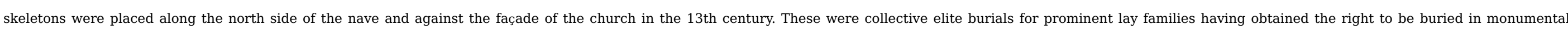
structures located against the church walls (Santiago-Rodriguez et al., 2019; Ribolini et al., 2017; Fornaciari et al., 2016).

\subsection{Skeletal remains}

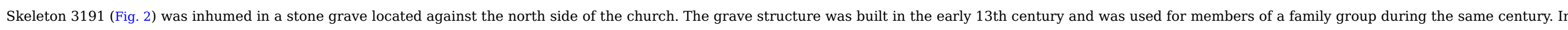

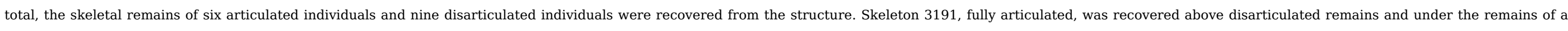

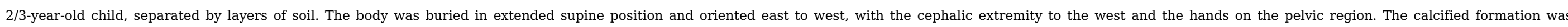
discovered in the thoraco-abdominal region, adjacent to the ninth and tenth right ribs (Fig. 2), where the liver is located.

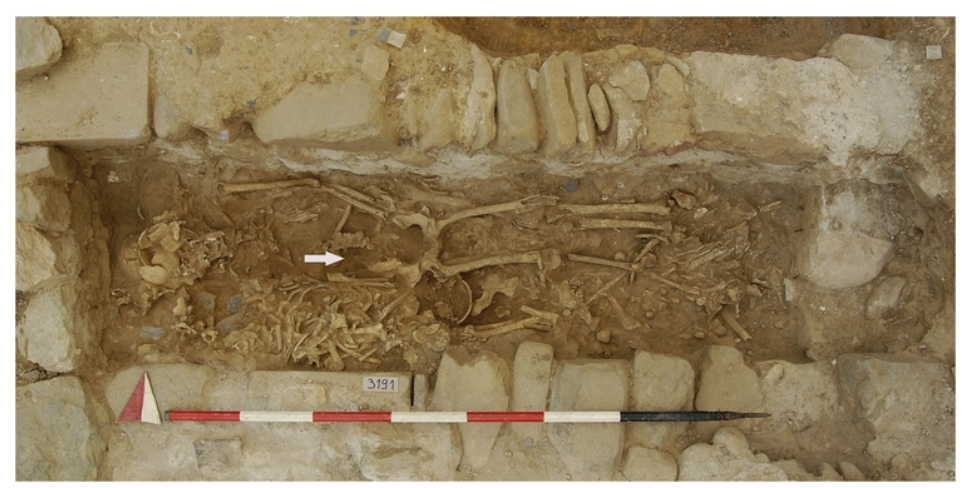

Fig. 2 13th century stone coffin associated with individual 3191. The white arrow indicates the location of the calcified formation.

\section{alt-ext: Fig. 2}

\subsection{Methods}

\subsubsection{Anthropology}

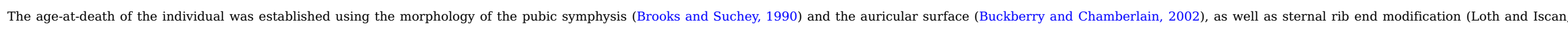

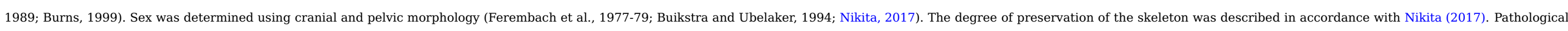
lesions were recorded using methods and standards set out in the Global History of Health Project (Steckel et al., 2005).

\subsubsection{Imaging}

Cone Beam Computed Tomography (CBCT) equipment (PlanMeca Promax Classic 3D) was used to obtain 3D (parameters 5.6-12 mAS with 86-85 kV), and 2D acquisition (parameters 10-10.5 mAS with 64 kV) images.

\subsubsection{Microscopic examination and chemical analysis}

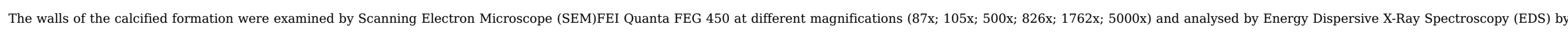

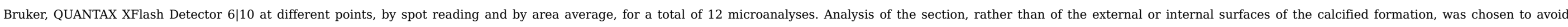
contamination with soil. 


\section{Results}

\subsection{Macroscopic examination}

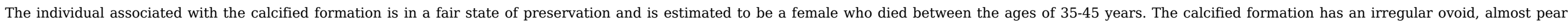

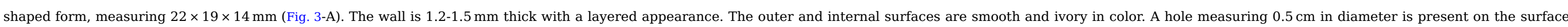

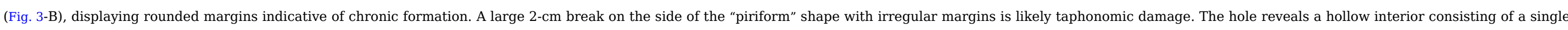
chamber with residual soil inside (Fig. 3-C). 


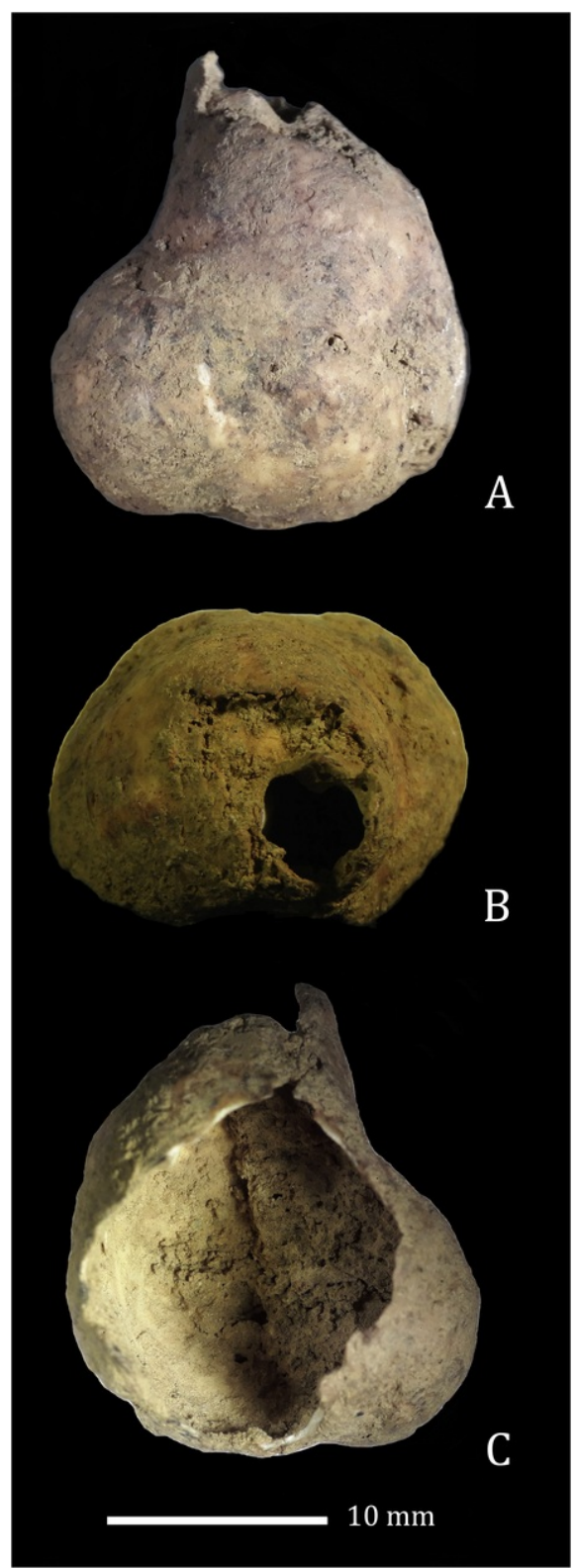

Fig. 3 Macroscopic image of the calcified formation: A) view of the intact side; B) view of the surface with a non-taphonomic hole; C) view of the side with taphonomic rupture alt-text: Fig. 3

\subsection{CBCT}

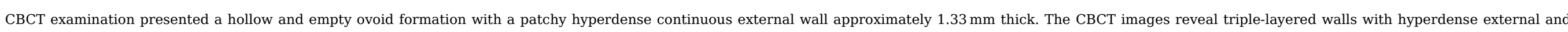
internal layers due to the high content of calcified tissue, while the middle layer is of lower density (Fig. 4). The volume of the formation is $3.06 \mathrm{~cm}^{3}$. 


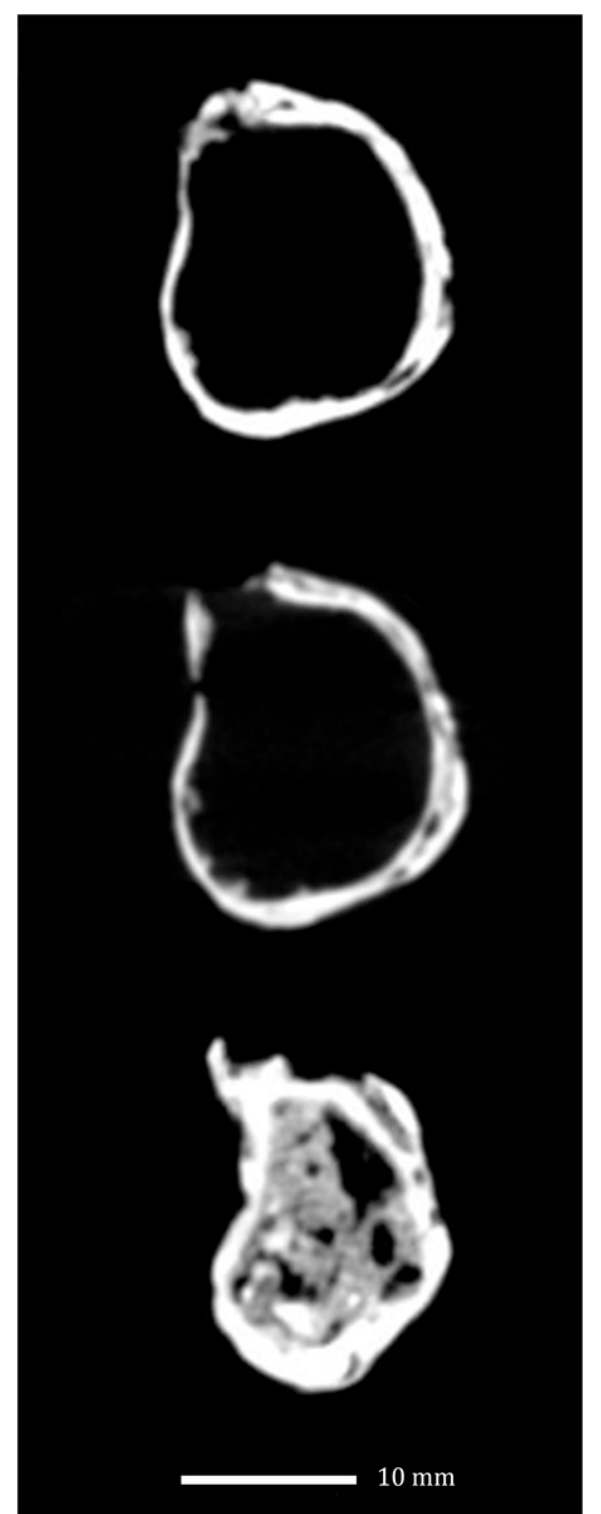

Fig. 4 CBCT images showing the triple-layered walls with hyperdense external and internal layers due to calcified tissue and the mid layer with lower density alt-text: Fig. 4

\subsection{Microscopic examination and chemical analysis}

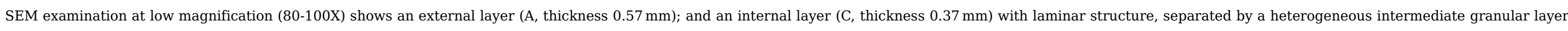

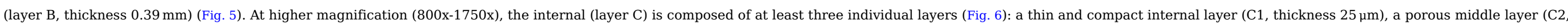
thickness $190 \mu \mathrm{m}$ ), and a compact external layer (C3, thickness $150 \mu \mathrm{m}$ ). 


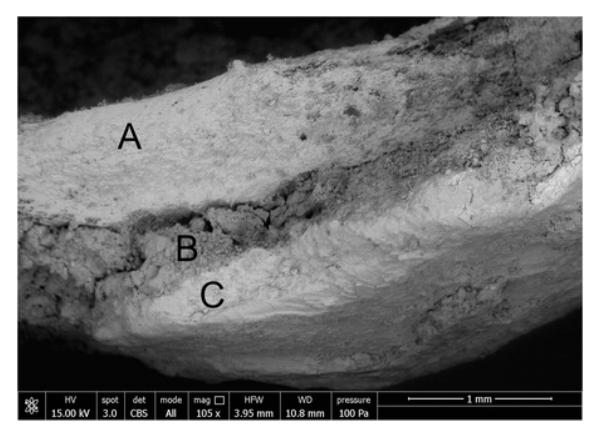

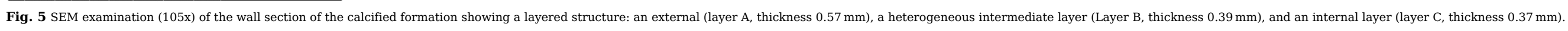
alt-text: Fig. 5

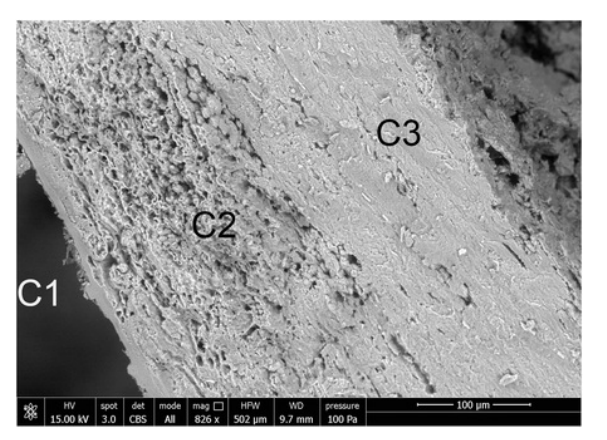

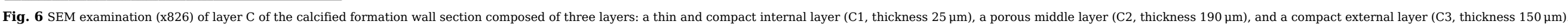
alt-text: Fig. 6

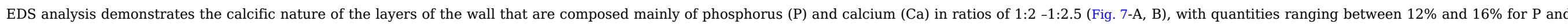

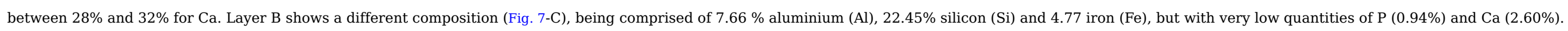



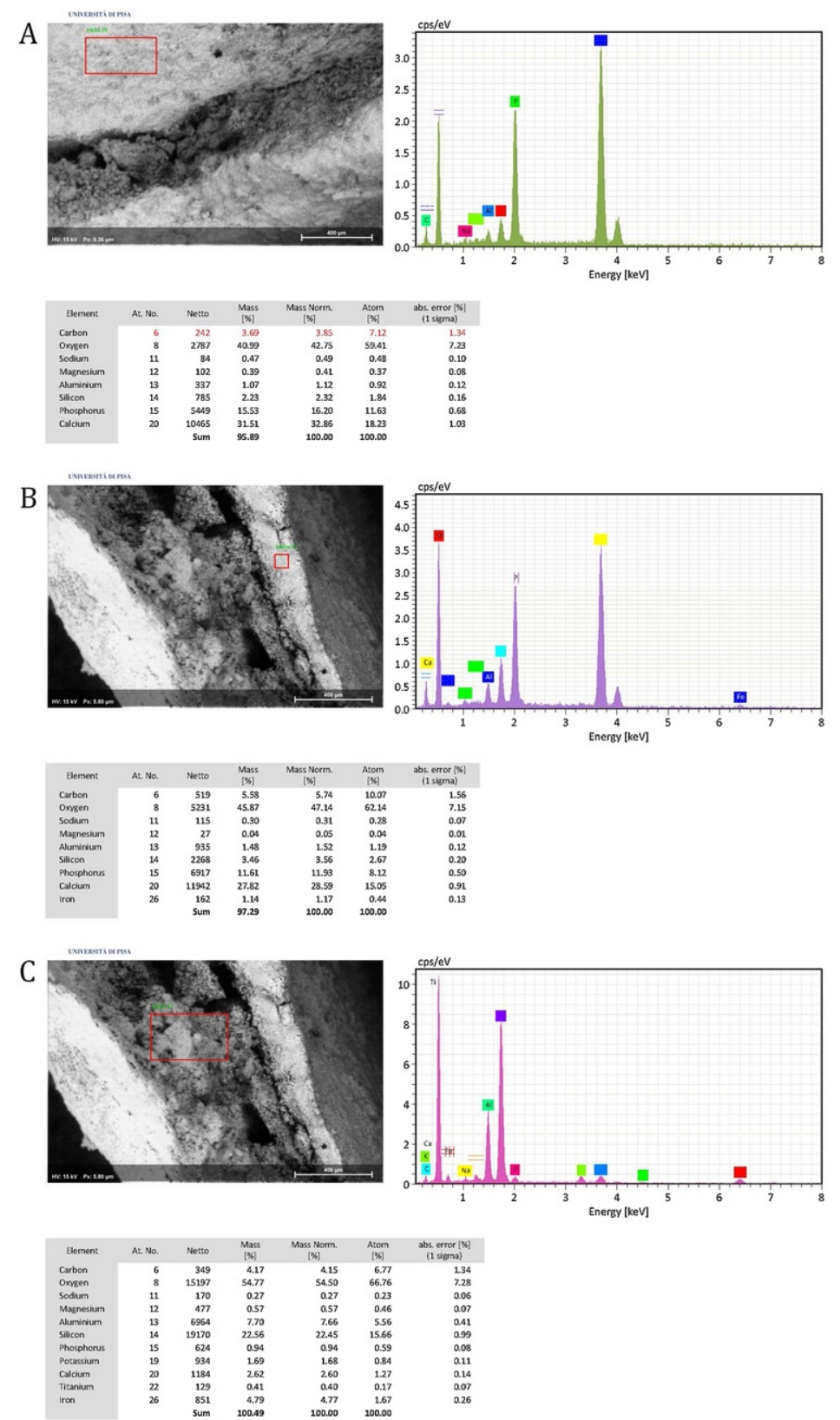

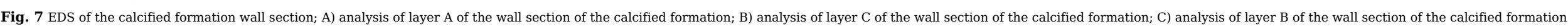
alt-text: Fig. 7

\section{Differential diagnosis}

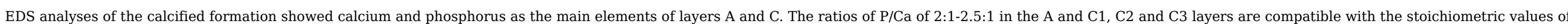




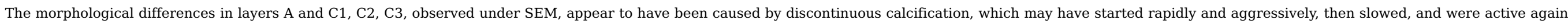
with increased intensity, possibly lasting for years (Kirsch, 2012).

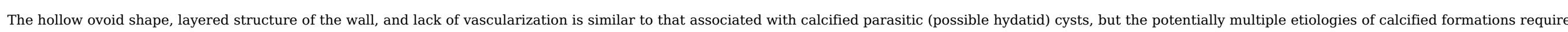

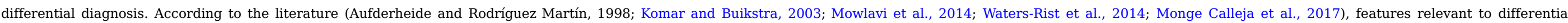
diagnosis include:

1 location in the body

2 size

3 shape

4 the presence of a chamber

5 appearance of the surface

6 the presence of vascularization

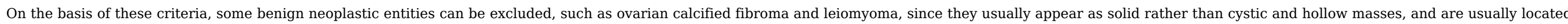

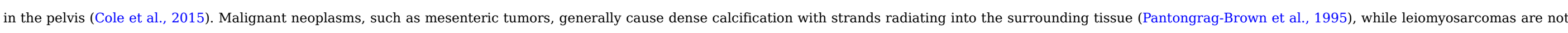

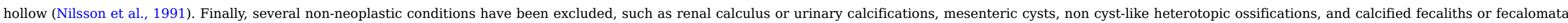
because their pattern is generally solid with a stone-like appearance (Kaya and Eris, 2011; Komar and Buikstra, 2003).

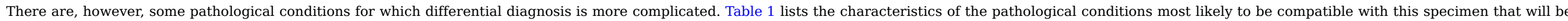
discussed further.

Table 1 List of characteristics considered in the differential diagnosis of the calcified formation.

\section{alt-text: Table 1}

\begin{tabular}{|c|c|c|c|c|c|c|c|c|c|c|}
\hline & \multirow[b]{2}{*}{ Present specimen } & \multicolumn{3}{|c|}{ Neoplastic diseases } & \multicolumn{2}{|c|}{ Non-neoplastic diseases } & \multicolumn{2}{|c|}{$\begin{array}{c}\text { Inflammatory/infectious } \\
\text { diseases }\end{array}$} & \multicolumn{2}{|c|}{ Parasitic diseases } \\
\hline & & Lymphoma & Teratoma & Renal cyst & $\begin{array}{c}\text { Functional ovarian } \\
\text { cyst }\end{array}$ & $\begin{array}{c}\text { Calcified } \\
\text { amputated ovary }\end{array}$ & Sarcoidosis & Tuberculosis & Cysticercosis & Echinococcosis \\
\hline Size & $2.2 \times 1.9 \times 1.4 \mathrm{~cm}$ & $<2-3 \mathrm{~cm}$ & $>6 \mathrm{~cm}$ & $2.5-6 \mathrm{~cm}$ & $0.5-2.5 \mathrm{~cm}$ & $<4 \mathrm{~cm}$ & $<3 \mathrm{~cm}$ & $<3 \mathrm{~cm}$ & $<2 \mathrm{~cm}$ & $1-10 \mathrm{~cm}$ \\
\hline Site & Pelvic, unilateral & $\begin{array}{l}\text { Thoraco- } \\
\text { abdominal }\end{array}$ & Pelvic, often unilateral & $\begin{array}{l}\text { Abdominal, } \\
\text { unilateral }\end{array}$ & $\begin{array}{l}\text { Pelvic, often } \\
\text { unilateral }\end{array}$ & Pelvic, unilateral & $\begin{array}{l}\text { Thoracic, } \\
\text { bilateral }\end{array}$ & $\begin{array}{l}\text { Thoraco- } \\
\text { abdominal }\end{array}$ & $\begin{array}{l}\text { Head; thoraco- } \\
\text { abdominal, limb }\end{array}$ & $\begin{array}{l}\text { Thoraco- } \\
\text { abdominal }\end{array}$ \\
\hline Mean age & $35-45$ years & $\begin{array}{l}\text { Subadult and } \\
\text { adulthood }\end{array}$ & $\begin{array}{l}\text { First } 6 \text { months to early } \\
\text { adulthood }\end{array}$ & $>50$ years & $\begin{array}{l}\text { Adulthood, } \\
\text { postmenopausal }\end{array}$ & Subadult & $45-60$ years & Peak 25-40 & $15-45$ years & Adulthood \\
\hline Shape & Ovoid & $\begin{array}{l}\text { Rounded, } \\
\text { punctate, linear }\end{array}$ & Rounded & Irregular & Irregularly ovoid & Curvilinear & $\begin{array}{l}\text { Irregularly } \\
\text { ovoid }\end{array}$ & $\begin{array}{l}\text { Irregularly } \\
\text { ovoid }\end{array}$ & Cigar-shaped & $\begin{array}{l}\text { Ovoid or } \\
\text { subspherical }\end{array}$ \\
\hline Aspect & Unilocular & Unilocular & $\begin{array}{l}\text { Unilocular or } \\
\text { multilocular }\end{array}$ & Often lobulated & Unilocular & Unilocular & Unilocular & Unilocular & Rice grain & $\begin{array}{l}\text { Unilocular or } \\
\text { multilocular }\end{array}$ \\
\hline Surface & Smooth & Coarse & Smooth & Inhomogeneous & Gritty & Inhomogeneous & Coarse & Coarse & Coarse & Smooth \\
\hline $\begin{array}{l}\text { Internal } \\
\text { content }\end{array}$ & Empty & Empty & Heterotopic material & Empty & Empty & Empty & Empty & Empty & Usually full & Empty or septa \\
\hline
\end{tabular}




\begin{tabular}{|c|c|c|c|c|c|c|c|c|c|c|}
\hline Vascularization & No & Yes & Yes & No & Scarse & Yes & Yes & Yes & No & No \\
\hline $\begin{array}{l}\text { Number of } \\
\text { lesions }\end{array}$ & Single & $\begin{array}{l}\text { Single or } \\
\text { multiple }\end{array}$ & Single & Single & Single & Single & $\begin{array}{l}\text { Multiple } \\
\text { (mean 20) }\end{array}$ & Multiple & Multiple & Often single \\
\hline
\end{tabular}

\subsection{Neoplastic diseases}

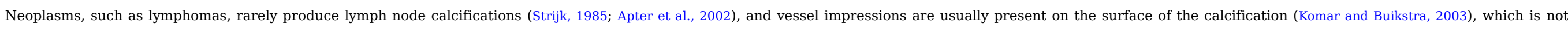

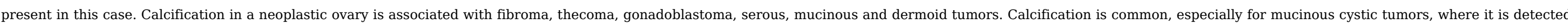

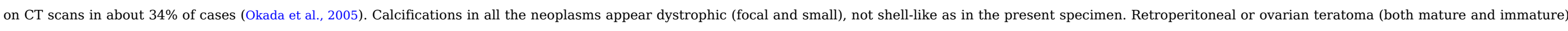

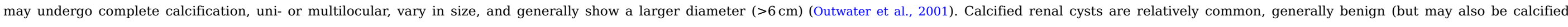

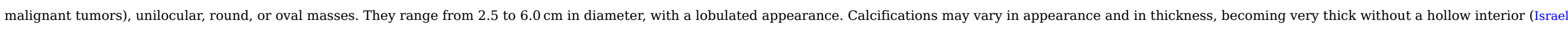
and Bosniak, 2003).

\subsection{Non-neoplastic diseases}

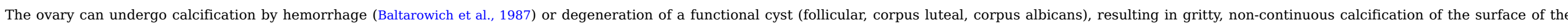

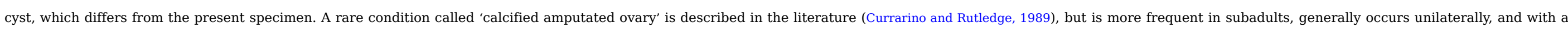

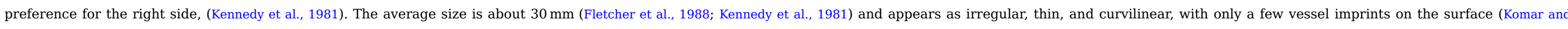
Buikstra, 2003). For these reasons, the diagnosis of calcific amputated ovary cannot be rejected, even if the location is not compatible with the specimen described here.

\subsection{Inflammatory/infectious diseases}

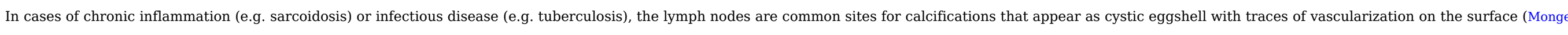

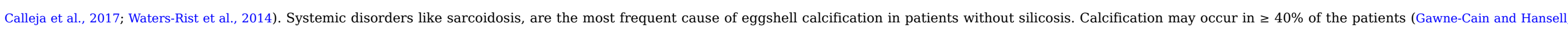

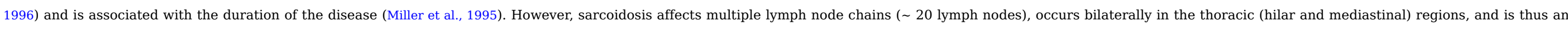
unlikely diagnosis in the present case.

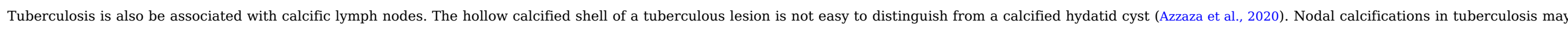

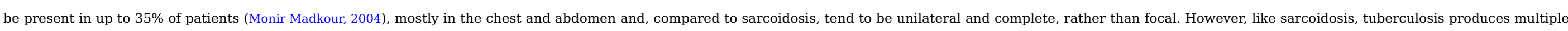
calcifications rather than a single lesion.

\subsection{Parasitic diseases}

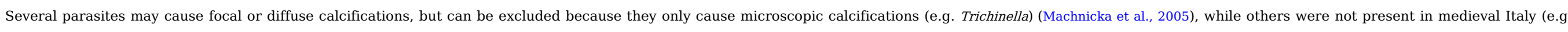
Dracunculus medinensis) (Gaeta et al., 2017).

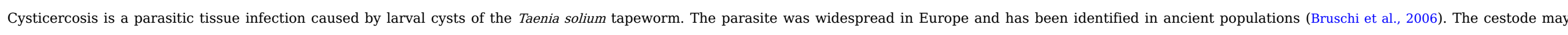

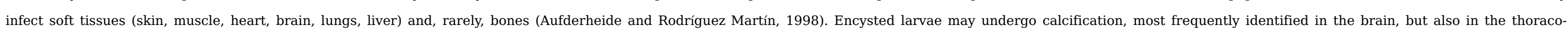

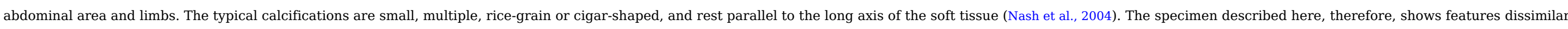
to those described for cysticercosis.

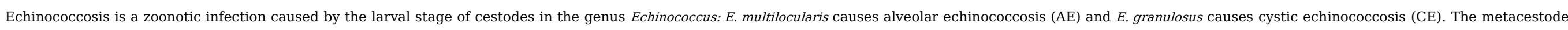

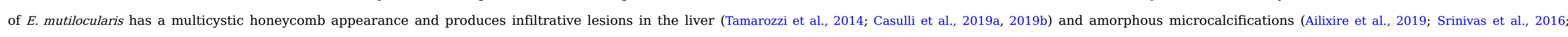
Eisenmberg, 2009; Pawlowski et al., 2001). AE is diffuse in central Europe, but not in peninsular Italy (Deplazes et al., 2017; Guerra et al., 2014). 


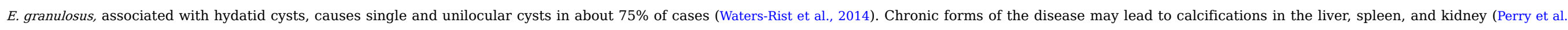

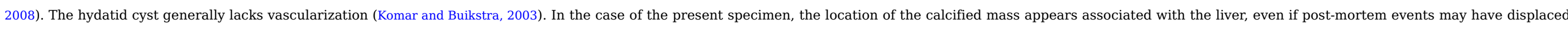

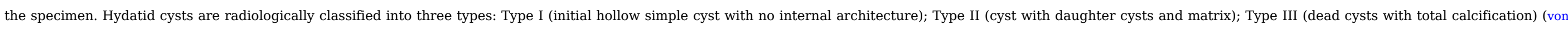
Sinner et al., 1991). The specimen from skeleton 3191 appears to be typical of a Type III (calcified) hydatid cyst.

\section{Discussion}

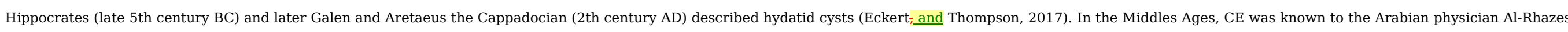

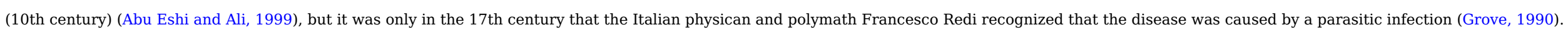

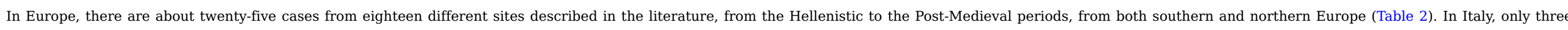

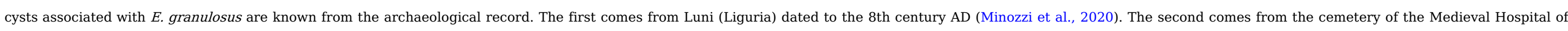

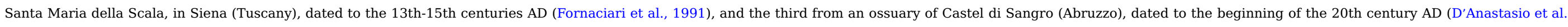

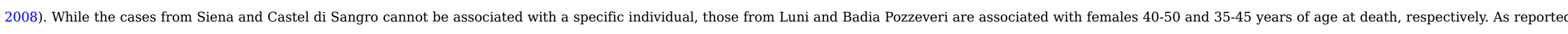

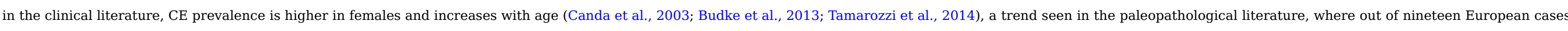

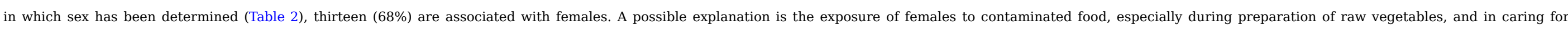
domestic dogs. These behaviors are believed to be the cause of the higher incidence of EC in women in developing countries (Budke et al., 2013).

Table 2 List of published European palaeopathological cases of hydatid cysts

\begin{tabular}{|c|c|c|c|c|c|c|}
\hline Site & Archaeological context & Chronology & Individual sex and age & $\begin{array}{l}\text { Number of } \\
\text { cystys }\end{array}$ & Localisation & Reference \\
\hline Siena, Tuscany (Italy) & Urban hospital cemetery & $1257-1499$ years & ND & 1 & ND & Fornaciari et al., 1991 \\
\hline Badia Pozzeveri, Tuseany (Italy) & Monastic site cemetery & 13th century & Female, 34-44y & 4 & thoraco abdominal & Fornaciari et al., 2019 \\
\hline Luni, La Spezia, Liguria (Italy) & Urban cemetery & 8th century & Female, $40-50$ y & 1 & thoraco-abdominal & Minozzi et al., 2020 \\
\hline Castel di Sangro, Abruzzo (Italy) & Urban cemetery & early 20th century & ND & 1 & ND & D’Anastasio et al., 2008 \\
\hline Amiens, Somme, (France) & Urban necropolis & 3rd-4th centuries & Sex unknown, adolescent & 2 & thoraco-abdominal & Mowlavi et al., 2014 \\
\hline Lunel-Viel, Hérault (France) & Rural cemetery & early Middle Ages & $2-4$ y & 1 & ND & Baud and Kramar, 1990 \\
\hline Saint-Prex, Vaude (Switzerland) & Rural cemetery & 6th-18th centuries & Male, 60 у са. & 1 & ND & $\begin{array}{l}\text { Baud and Kramar, 1990; Kramar, } \\
1996\end{array}$ \\
\hline Prádena del Rincón, Madrid (Spain) & Rural cemetery & $\begin{array}{l}\text { 12th-13th } \\
\text { centuries }\end{array}$ & Female, $25-39$ y & 1 & pelvic & Monge Calleja et al., 2017 \\
\hline Pydna, Macedonia (Greece) & Urban necropolis & 3rd century BC & Female? , 18-30 y? & 1 & ND & Antikas and Winn Antikas, 2016 \\
\hline Sankt Jørgens Spital, Naestved (Denmark) & Leper hospital cemetery & 1450 year c. & Female, 16 y ca. & $>72$ & abdominal and pelvic & Weiss and Møller-Christensen, 1971 \\
\hline Skriðuklaustur, Fljótsdalur (Iceland) & $\begin{array}{l}\text { Monastic site/hospital } \\
\text { cemetery }\end{array}$ & 1493-1554 years & $\begin{array}{l}4 \text { females, } 3 \text { males, } 1 \mathrm{ND}, \\
>50 \mathrm{y}\end{array}$ & $>21$ & thoraco-abdominal & Kristjánsdóttir and Collins, 2010 \\
\hline Dziekanowice, Wielkopolska (Poland) & Rural cemetery & 1050-1200 years & Female, adult & 2 & $\begin{array}{l}\text { thoraco-abdominal and } \\
\text { femoral }\end{array}$ & Gładykowska-Rzeczycka et al., 2003 \\
\hline Caherquin, Munster (Ireland) & Rural cemetery & $\begin{array}{l}\text { 15th-17th } \\
\text { centuries }\end{array}$ & Female, adult & 1 & thoraco-abdominal & Power, 2010 \\
\hline
\end{tabular}




\begin{tabular}{|c|c|c|c|c|c|c|}
\hline Cork, Munster (Ireland) & Urban cemetery & centuries & Female, 45 у са. & 1 & thoraco-abdominal & Power, 1997, 2010 \\
\hline Moorabbey, Munster (Ireland) & Monastic site cemetery & $\begin{array}{l}\text { 18th-19th } \\
\text { centuries }\end{array}$ & Male, adult & 1 & thoraco-abdominal & Power, 2010 \\
\hline Winchester, Hampshire (England) & Rural cemetery & early medieval & Male, adult & 1 & abdominal & Price, 1975 Wells and Dallas, 1976 \\
\hline $\begin{array}{l}\text { Orton Longueville, Cambridgeshire } \\
\text { (England) }\end{array}$ & Rural cemetery & 2nd century BC & Female, 45 у са. & 1 & thoracic & Wells and Dallas, 1976 \\
\hline Ensi, Hebrides (Scotland) & Rural cemetery & 17th century & 1 & 1 & ? & Price, 1975 Wells and Dallas, 1976 \\
\hline Orkley (Scotland) & ? & $?$ & $?$ & 1 & ? & Brothwell, 1978 \\
\hline
\end{tabular}

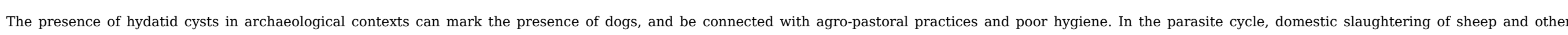

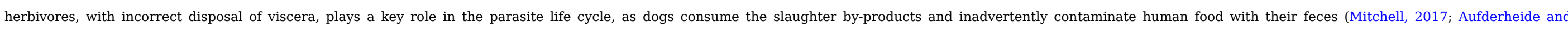
Rodríguez-Martín, 1998; Reinhard, 1992). Today, the disease is more prevalent in places where domestic animals (cattle, sheep, and dogs) and humans live in close contact (Possenti et al., 2016).

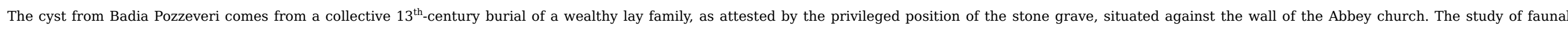

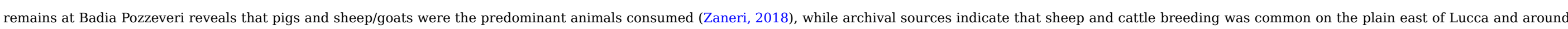
Lake Bientina (Seghieri, 1978).

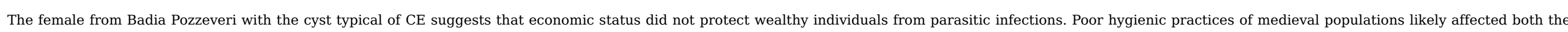

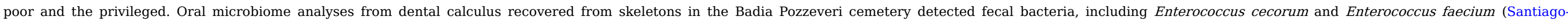

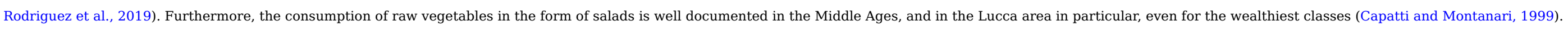

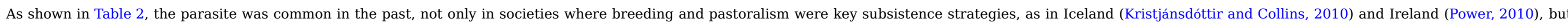

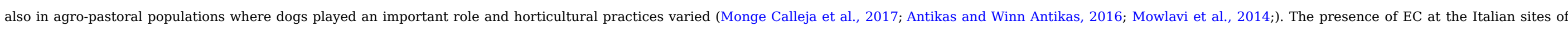

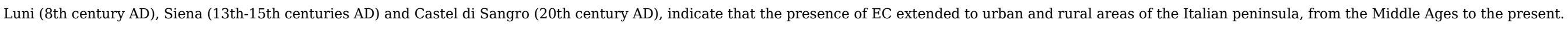

\section{Conclusions}

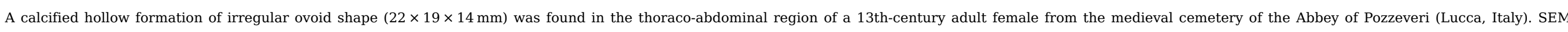

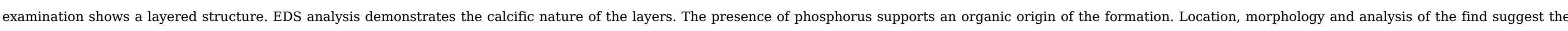

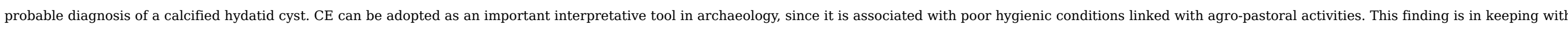

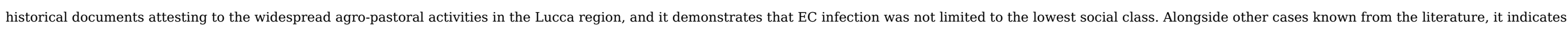
a widespread presence of the parasite Echinococcus granulosus in central Italy in the Middle Ages.

\section{Uncited references}

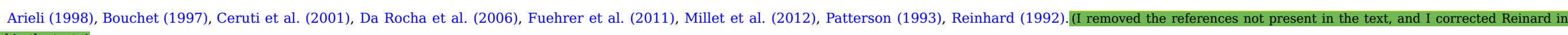

\section{Reinhard in the text.}

\section{Declaration of Competing Interest}

The authors report no declarations of interest.

\section{Acknowledgements}

The authors are grateful to Professor Gino Fornaciari (University of Pisa) for his revision of the text and to Dr. Laura Cignoni for the linguistic revision of the manuscript.

This research did not receive any specific grant from funding agencies in the public, commercial, or not-for-profit sectors. 


\section{References}

Abu Eshi S. and Ali M.E, Hydatid cyst associated with pregnancy: A case report and review of the literature, Ann. Saudu. Med. 19, 1999, 130-131, https://doi.org/10.5144/0256-4947.1999.130.

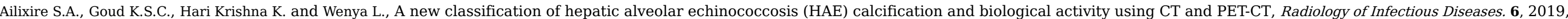
61-67, https://doi.org/10.1016/j.jrid.2019.06.002.

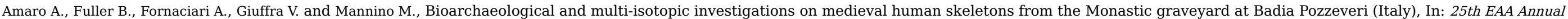
Meeting (Bern, 2019) - Abstract Book. Bern, 2019, p. 380.

Antikas T.G. and Winn Antikas L.K., Hydatidosis in a pregnant woman of the $3^{\text {rd }}$ century BC, Greece, Int. J. Osteoarchaeol. 26, 2016, 920-924, https://doi.org/10.1002/oa.2475.

Apter S., Avigdor A., Gayer G., Portnoy O., Zissin R. and Hertz M., Calcification in lymphoma occurring before therapy: CT features and clinical correlation, Am. J. Roentgenol. 178, 2002, 935-938,

https://doi.org/10.2214/ajr.178.4.1780935

Arieli R., Human remains from the Har Hazofim observatory Tembs (Mt. Scopus, Jerusalem), Anticot. 35, 1998, 3742 .

Aufderheide A.C. and Rodríguez-Martín C., The Cambridge Encyclopedia of Human Paleopathology, 1998, Cambridge University Press; Cambridge.

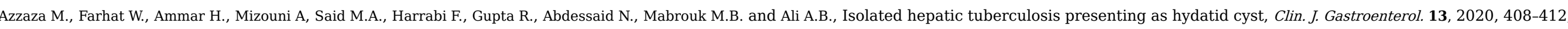

https://doi.org/10.1007/s12328-019-01071-w.

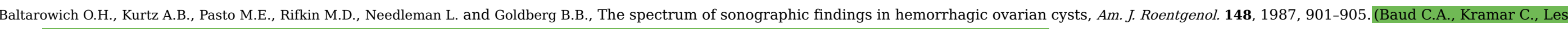
calcifications biologiques en archéologie, Bulletins et Mémoires de la Société d’anthropologie de Paris, Nouvelle Séries 2, 1990, 163-169.)

Bouchet F., Intestinal eapillariasis in neolithic inhabitants of Chalain (Jura, France), Laneet 349, 1997, 256, https.//doi.org/10.1016/S0140-6736(05)64868-4.

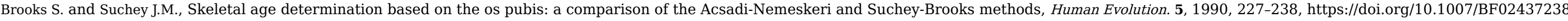

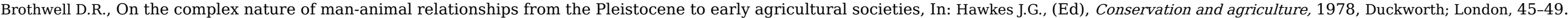

Bruschi F., Masetti M., Locci M.T., Ciranni R. and Fornaciari G., Short report: cysticercosis in an Egyptian mummy of the late Ptolemaic period, Am. J. Trop. Med. Hyg. 74, 2006, 598-599.

Buckberry J.L. and Chamberlain A.T., Age estimation from the auricular surface of the ilium: a revised method, Am. J. Phys. Anthropol. 119, 2002, 231-239, https://doi.org/10.1002/ajpa.10130.

Budke C.M., Deplazes P. and Torgerson P.R., Global socioeconomic impact of cystic echinococcosis, Emerg. Infect. Dis. 12, 2006, 296-303, https://doi.org/10.3201/eid1202.050499.

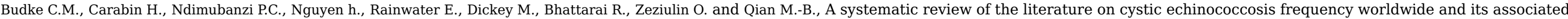

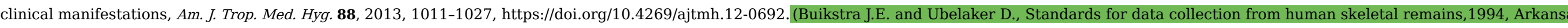
Archaeological Survey Report 44; Fayetteville, Arkansas.)

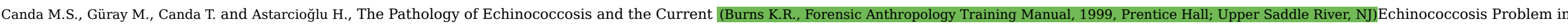

Western Turkey (A Report of Pathologic Features in 80 Cases), Turk. J. Med. Sci. 33, 2003, 369-374.

Capatti A. and Montanari M., La cucina italiana. Storia di una cultura, 1999, Editori Laterza; Roma-Bari.

Casulli A., Barth T.F.E. and Tamarozzi F, Echinococcus multilocularis, Trends Parasitol. 35, 2019a, 738-739, https://doi.org/10.1016/j.pt.2019.05.005.

Casulli A., Siles-Lucas M. and Tamarozzi F., Echinococcus granulosus sensu lato, Trends Parasitol. 35, 2019b, 663-664, https://doi.org/10.1016/j.pt.2019.05.006.

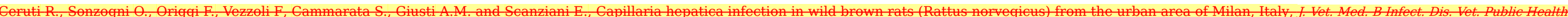

48, 2001, 235-240, https.//dei.org/10.1046/j.1439-0450.2001.00436.x 
Clinton White A. and Weller P.F, Cestodes, Harrison's Principles of Internal Medicine, 17th edn, 2008, McGraw-Hill Professional; New York,, $1336-1341$.

Cole G., Rando C., Sibun L. and Waldron T., Case report: A giant calcified uterus, likely due to benign leiomyoma, Int. J. Paleopathol. 10, 2015, 51-57, https://doi.org/10.1016/j.ijpp.2015.05.003.

Currarino G. and Rutledge J.C., Ovarian torsion and amputation resulting in partially calcified, pedunculated cystic mass, Pediatr. Radiol. 19, 1989, 395-399, https://doi.org/10.1007/BF02387636.

D’Anastasio R., Vitullo G., Paolucci A. and Michetti E., A paleopathological case of Echinococcus cyst, J. Paleopathol. 20, 2008, 67-73.

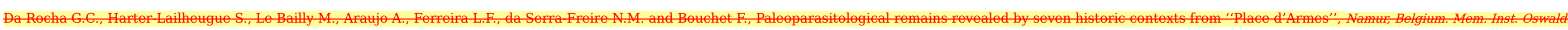
Ertz. 101, 2006, 43-52, https.//doi.org/10.1590/s0074 02762006001000008.

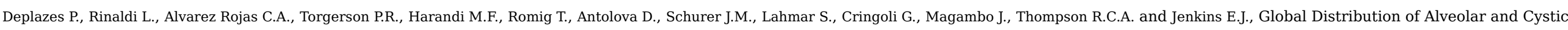
Echinococcosis, In: Thompson R.C.A., Deplazes P. and Lymbery A.J., (Eds.), Advances in Parasitology 95, 2017, Academic Press, 315-493, https://doi.org/10.1016/bs.apar.2016.11.001.

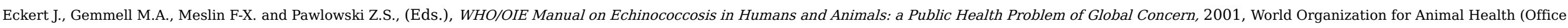
International des Epizooties) and World Health Organization; Geneva.

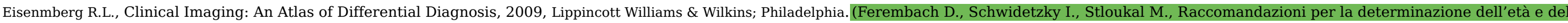
sesso sullo scheletro, Rivista di Antropologia 60,1977-79, 5-51.)

Fletcher R.M., Boal D.K.B., Karl S.R. and Gross G.W., Ovarian torsion: a usual case of bilateral pelvic calcifications, Pediatr. Radiol. 18, 1988, $172-173$, https://doi.org/10.1007/BF02387567.

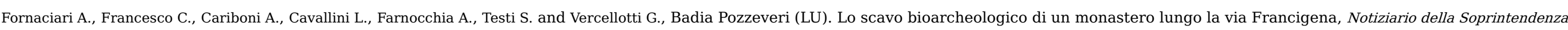
Archeologica della Toscana 11, 2016, 123-135.

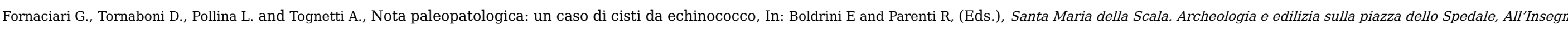
del Giglio, Firenze, 1991, 443-445.

Fuehrer H.P., Igel P. and Auer H., Capillaria hepatiea in man an overvie of hepatic capillariosis and spurious infections, Parasitol. Res. 109, $2011,969-979$, https://doi.org/10.1007/s00436 011 2494 1.

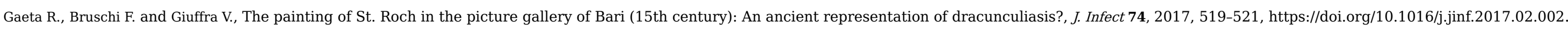

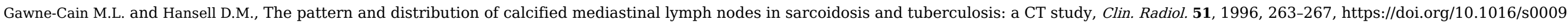
9260(96)80343-6.

Gładykowska-Rzeczycka J.J., An echinococcosis cyst from an early Medieval cemetery in Poland, Tc, histological, and DNA findings, J. Paleopathol. 15, 2003 , 47-58.

Grove D.I., A history of human helminthology, 1990, CAB International; Wallingford.

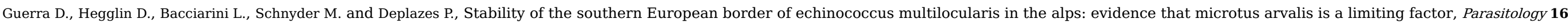
2014, 1-10, https://doi.org/10.1017/S0031182014000730.

Israel G.M. and Bosniak M.A., Calcification in Cystic Renal Masses: Is It Important in Diagnosis?, Radiology. 226, 2003, 47-52, https://doi.org/10.1148/radiol.2261011704.

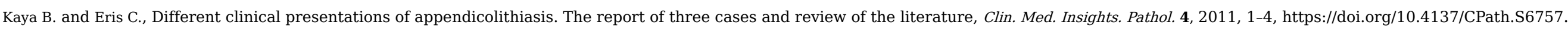
Kennedy L.A., Pinckney L.E., Currarino G. and Votteler T.P., Amputated Calcified Ovaries in Children, Radiology. 141, 1981, 83-86, https://doi.org/10.1148/radiology.141.1.7291547.

Kirsch T., Biomineralization-An Active or Passive Process?, Connective Tissue Research. 53, 2012, 438-445, https://doi.org/10.3109/03008207.2012.730081.

Komar D. and Buikstra J.E., Differential diagnosis of a Prehistoric biological object from the Koster (Illinois) Site, Int. J. Osteoarchaol. 13, 2003, 157-164, https://doi.org/10.1002/oa.670. 
Kristjánsdóttir S. and Collins C., Cases of hydatid disease in medieval Iceland, Int. J. Osteoatchaeol. 21, 2010, 479-486, https://doi.org/10.1002/oa.1155.

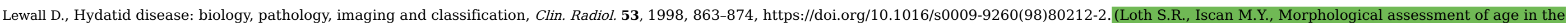

\section{adult: the thoracic region, in: Iscan M.Y. (Ed.), Age markers in the human skeleton, 1989, Charles C. Thomas; Springfield, 105-135.)}

Machnicka B., Dziemian E., Dabrowska J. and Walski M., Calcification of Trichinella spiralis larval capsule, Parasitol. Res. 97, 2005, 501-504, https://doi.org/10.1007/s00436-005-1465-9.

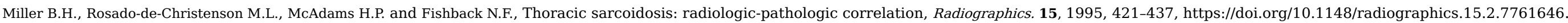

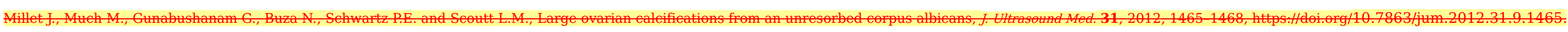

Minozzi S., De Sanctis M., Isack R., Caramella D., Gervasini L. and Giuffra V., Un antico caso di parassitosi zoonotica da Luni (SP), Medicina Historica 4 (Suppl. 1), 2020 , $114-115$.

Mitchell P.D., Human parasites in the Roman World: health consequences of conquering an empire, Parasitology. 144, 2017, 48-58, https://doi.org/10.1017/S0031182015001651.

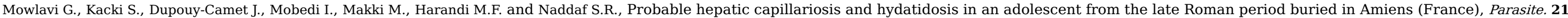
2014, 9, https://doi.org/10.1051/parasite/2014010.

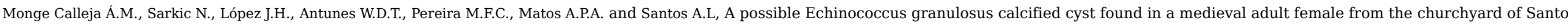
Domingo de Silos (Prádena del Rincón, Madrid, Spain), Int. J. Paleopathol. 16, 2017, 5-13, https://doi.org/10.1016/j.jpp.2017.01.005.

Monir Madkour M., Tuberculosis, 2004, Springer-Verlag; Berlin.

Moro P.L., Gilman R.H., Verastegui M., Bern C., Silva B. and Bonilla J.J., Human hydatidosis in the central Andes of Peru: evolution of the disease over 3 years, Clin. Infect. Dis. 29, 1999, 807-812,

https://doi.org/10.1086/520440.

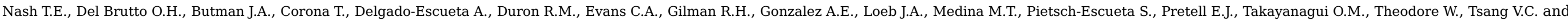
Garcia H.H., Calcific neurocysticercosis and epileptogenesis, Neurology. 62, 2004, 1934-1938, https://doi.org/10.1212/01.WNL.0000129481.12067.06.

Nikita E., Osteoarchaeology. A Guide to the macroscopic study of human skeletal remains, 2017, Academic Press; San Diego.

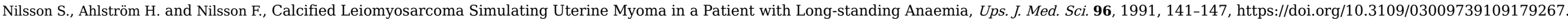

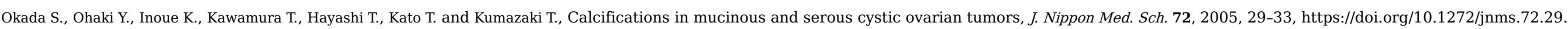

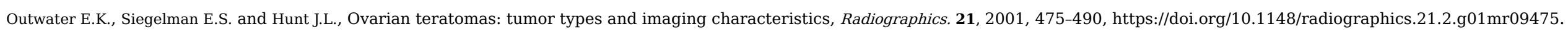

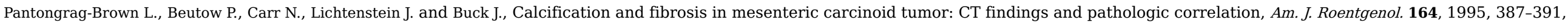

https://doi.org/10.2214/ajr.164.2.7839976.

Patterson K.D., Echineosis (Hydatidosis), In: Kiple K.F., (Ed), The Gambridge World History of Human Disease, 1993, Gambridge University Press, New York, p. 703.

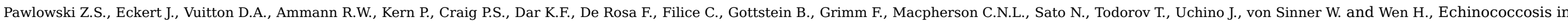

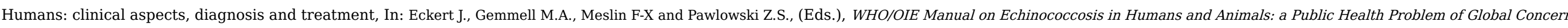
2001, World Organization for Animal Health (Office International des Epizooties) and World Health Organization; Geneva, 20-71.

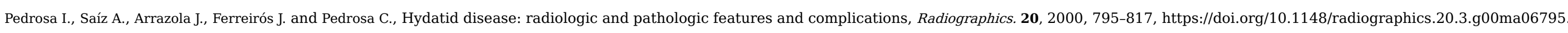

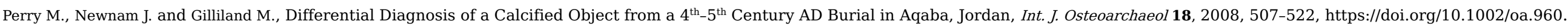

Polat P., Kantarci M., Alper F., Suma S., Koruyucu M.B. and Okur A., Hydatid disease from head to toe, Radiographics. 23, 2003, 475-494, https://doi.org/10.1148/rg.232025704. 


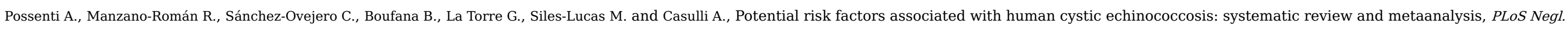
Trop. Dis. 10, 2016, , e0005114https://doi.org/10.1371/journal.pntd.0005114.

Power C., The Human Remains from 19-20 Cove Street, Cork City, The Journal of the Cork Historical and Archaeological Society. 102, $1997,79-88$.

Power C., Medieval tapeworm infestation, J. Kerry Archaeol. Hist. Soc. 10, 2010, 22-32, Price, 1975.

Reinhard K.J., Parasitology as an interpretative tool in archaeology, American Antiquity 57, 1992, 232-245 https://digitalcommons.unl.edu/natresreinhard/33/.

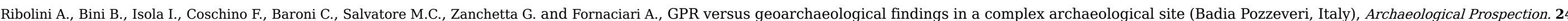
2017, 141-156, https://doi.org/10.1002/arp.1561.

Romig T., Zeyhle E., Macpherson C.N., Rees P.H. and Were J.B., Cyst growth and spontaneous cure in hydatid disease, Lancet. 1, 1986, 861, https://doi.org/10.1016/s0140-6736(86)90974-8.

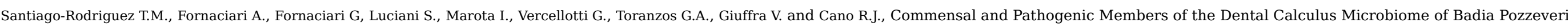
Individuals from the 11th to 19th Centuries, Genes 10, 2019, https://doi.org/10.3390/genes10040299, pii: E299.

Seghieri M., Pozzeveri: una badia, 1978, Stamperia Benedetti; Pescia (Italy).

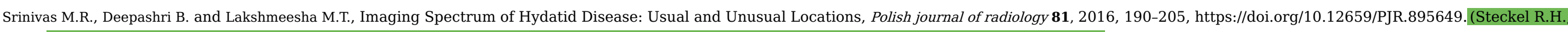
Larsen C.S., Sciulli P.W., Walker P.L., Data collection codebook. The global history of health project, 2005, The Ohio State University; Columbus.

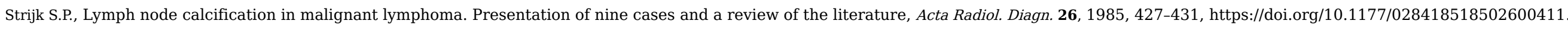

Tamarozzi F., Brunetti E. and Vuitton D.A., Echinococcosis, In: Bruschi F., (Ed), Helminth Infections and their Impact on Global Public Health, 2014, Springer-Verlag; Wien, 153-200.

Thompson R., Echinococcosis, In: Gillespie S. and Pearson R., (Eds.), Principles and Practice of Clinical Parasitology, 2001, John Wiley \& Sons; Chichester, 585-612.

von Sinner W., te Strake L., Clark D. and Sharif H., MR imaging of Hydatid Disease, AJR Am. J. Roentgenol 157, 1991, 741-745, https://doi.org/10.2214/ajr.157.4.1892028.

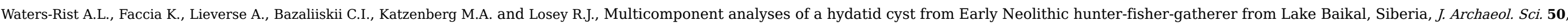
2014, 51-62, https://doi.org/10.1016/j.jas.2014.06.015.

Weiss D.L. and Møller-Christensen V., Leprosy, Echinococcosis and amulets: a study of a medieval Danish inhumation, Med. Hist. 15, 1971, 260-267, https://doi.org/10.1017/s0025727300016719.

Wells C. and Dallas C., Romano-British pathology, Antiquity. 50, 1976, 53-55, https://doi.org/10.1017/S0003598X00101760.

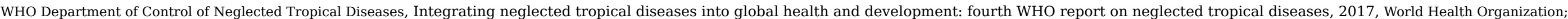
Geneva.

Zaneri T., Rural Production, Peasant Participation, and State Power: The Reshaping of Medieval Italy. Doctoral Dissertation, 2018, New York University.

\section{Queries and Answers}

Query: Please check the presentation of the affiliations and the country name added to the affiliations, and correct if necessary.

Answer: affiliations are correct

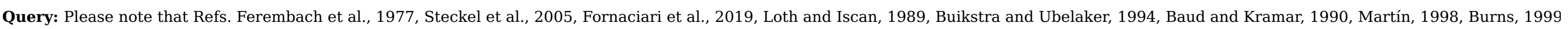

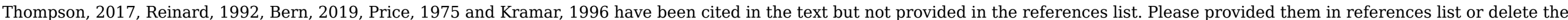
citations from the text.

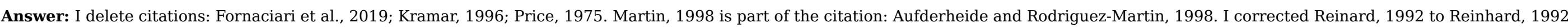


